
well as reproducibility evaluation. Three sensors (AE51, Cairclip and Canarin) were retained to

\title{
A methodology for the characterization of portable sensors for air quality measure with the goal of deployment in citizen science
}

\author{
Baptiste Languille $^{1 *}$, Valérie Gros ${ }^{1}$, Nicolas Bonnaire ${ }^{1}$, Clément Pommier ${ }^{1}$, \\ Cécile Honoré $^{2 \dagger}$, Christophe Debert ${ }^{2}$, Laurent Gauvin ${ }^{2}$, Salim Srairi ${ }^{3}$, Isabella \\ Annesi-Maesano ${ }^{4}$, Basile Chaix ${ }^{5}$, Karine Zeitouni6 \\ ${ }^{1}$ Laboratoire des sciences du climat et de l'environnement CNRS-CEA-UVSQ, IPSL, Gif-Sur-Yvette, \\ France. \\ ${ }^{2}$ Airparif, Paris, France. \\ ${ }^{\dagger}$ Now at: Mairie de Paris, Direction de la voirie et des déplacements, Paris, France \\ ${ }^{3}$ CEREMA, Trappes-en-Yvelines, France. \\ ${ }^{4}$ EPAR, IPLESP, INSERM et Sorbonne université, Faculté de médecine Saint-Antoine, Paris, France. \\ IINSERM, Sorbonne université, institut Pierre Louis d'épidémiologie et de santé publique IPLESP, \\ Nemesis team, Paris, France. \\ ${ }^{6}$ Université de Versailles Saint-Quentin, Versailles, France.
}

\section{Abstract}

The field of small air quality sensors is of growing interest within the scientific community, especially because this new technology is liable to improve air pollutant monitoring as well as be used for personal exposure quantification. Amongst the myriad existing devices, the performances are highly variable; this is why the sensors must be rigorously assessed before deployment, according to the intended use. This study is included in the Polluscope project; its purpose is to quantify personal exposure to air pollutants by using portable sensors. This paper designs and applies a methodology for the evaluation of portable air quality sensors to eight devices measuring $\mathrm{PM}, \mathrm{BC}, \mathrm{NO}_{2}$ and $\mathrm{O}_{3}$. The dedicated testing protocol includes static ambient air measurements compared with reference instruments, controlled chamber and mobility tests, as be used for the field campaigns. The reliability of their performances were robustly quantified by

\footnotetext{
${ }^{*}$ Corresponding author. Tel: +33169083263

E-mail address: baptiste.languille@1sce.ipsl.fr
} 
28 using several metrics. These three devices (for a total of 36 units) were deployed to be worn by

29 volunteers for a week. The results show the ability of sensors to discriminate between different

30 environments (i.e., cooking, commuting or in an office). This work demonstrates, first, the ability

31 of the three selected sensors to deliver data reliable enough to enable personal exposure

32 estimations, and second, the robustness of this testing methodology.

33

34 Keywords: Paris region; Black carbon; Nitrogen oxides; Particulate matter; Personal exposure, 35 Mobile measurements.

36 


\section{INTRODUCTION}

39 Atmospheric pollution is a well-identified threat to health (WHO, 2003; IARC, 2013; Sante

40 Publique France, 2016; European Environment Agency, 2017). WHO (2014) indicates that 94\%

41 of world population is exposed to levels of air pollution that are hazardous. This highlights that monitoring pollution accurately is very important to understand better the phenomenon and to suggest solutions for its mitigation.

44 So far, monitoring networks do not enable the precise measurement of personal exposure to pollution, defined as the pollutant concentration inhaled by people over a period of time. The first reason is the interpolation between stations. Even with a great number of stations, interpolation is still required to quantify personal exposure, and that leads to errors. Second, the air sampling height (set by the legislation between 1.5 and 3 meters above ground level) is often above the average height of our respiratory system, which also induces a difference with the inhaled air.

50 Furthermore, monitoring stations maps and daily reports are only based on outdoor

51 measurements, but people spend most of their time indoors (Klepeis et al., 2001), where high

52 concentrations of air pollutants may exist (Adgate et al., 2004). This is why it is of primary 53 importance to measure also indoor concentrations and to take into account the different 54 environments where people live to quantify their personal exposure.

55 The solution to quantifying personal exposure could be portable air pollutant sensors. The field of 56 small sensors is constantly improving (Borghi et al., 2017) thanks to the progress of available 57 technologies. This type of sensor presents two main advantages over classical measurements. 58 First, the sensor units are small and thus easily worn all day long by people, which could enable 59 robust 24/7 personal exposure measurements (including indoor air measurements). Second, some 
60 of these sensor units are relatively low cost, which allows large numbers of units to be purchased

61 and enables simultaneous monitoring of a large number of places.

62 Conversely, the main drawback of these devices is their questionable accuracy. In the field of 63 small air quality sensors, several kinds of studies were published, such as sensor development 64 (Hu et al., 2016; Mead et al., 2013; Peng et al., 2013), sensor assessment (Lin et al., 2015; 65 Burkart et al., 2010; Sousan et al., 2016), exploratory measures of personal exposure (Velasco et 66 al., 2016; Velasco and Tan, 2016; Hu et al., 2014) and full-scale project involving large field 67 campaigns (Mead et al., 2013; Castell et al., 2015, 2017; Schneider et al., 2017; Hasenfratz et al., 68 2015).

69 Although previous projects were interesting, some limitations invite researchers to keep on 70 improving the methods to quantify personal exposure. First, it is highlighted that most of the 71 small sensors suffer from lower precision than reference instruments, which shows the 72 importance of a robust assessment prior to launching field campaigns. The report by Lewis et al. 73 (2018) (not yet published during the experiments presented below) gave the state of the art of the 74 low-cost air quality sensors. However, not all studies pushed the sensor characterization as far as 75 it should be. Second, personal exposure is about measuring air quality as close as possible to the 76 inhaled air, which is why it is of primary importance to give the sensor units directly to people. 77 But some studies used sensor units on static measurements or attached to vehicles (Velasco and 78 Tan, 2016; Castell et al., 2017; Deville Cavellin et al., 2016; Duvall et al., 2016; Fishbain et al., 79 2017; Gao et al., 2015; Holstius et al., 2014), which is not as relevant as asking people to 80 personally carry the sensor units. Finally, personal exposure is relevant in health impact studies 81 for which it is interesting to have several measured pollutants. This last consideration points out 82 single pollutant measurement studies as a limitation. 
83 Île-de-France is the Paris region, it is the most densely populated in France but with great

84 disparities between Paris centre and remote places. This region is also characterized as an

85 important atmospheric pollutant emission area. More than one million inhabitants are exposed to

86 nitrogen dioxide concentration exceeding the limit value (annual mean of $40 \mu \mathrm{g} \mathrm{m}^{-3}$ ) and $85 \%$

87 of the population is exposed to $\mathrm{PM}_{2.5}$ levels above the long-term objective ${ }^{1}$ (annual mean of $8810 \mu \mathrm{g} \mathrm{m}^{-3}$ ) (Airparif, 2017, 2018, 2019).

89 The project Polluscope funded by the French National Research Agency addresses precisely 90 the previously mentioned issues by asking volunteers to carry portable geolocalised sensor units 91 all day long during one week in order to quantify personal exposure to several pollutants. This 92 project is characterized by multidisciplinary objectives (large field campaigns with many 93 volunteers, a cloud platform for data processing, a big data analysis, an epidemiological study, a 94 deep data processing, etc.), but the first step was to conduct a robust sensor selection and 95 assessment.

96 Polluscope is also defined by the diversity of the studied pollutants as this project will monitor $97 \mathrm{PM}\left(\mathrm{PM}_{10}, \mathrm{PM}_{2.5}\right.$ and $\left.\mathrm{PM}_{1}\right)$, black carbon $(\mathrm{BC})$ and nitrogen dioxide $\left(\mathrm{NO}_{2}\right)$. The choice of the 98 monitored pollutants was made according to their impact on health as well as the exceedances 99 experienced in Île-de-France for each pollutant. Indeed, Airparif, the French agency for air 100 quality monitoring, states that some pollutants $\left(\mathrm{PM}_{10}, \mathrm{PM}_{2.5}, \mathrm{NO}_{2}, \mathrm{O}_{3}\right.$, benzene) still exceed the 101 limits. As these pollutants (as well as $\mathrm{BC}$, which is not regulated) have a positive deleterious

\footnotetext{
${ }^{1}$ Definitions of limit value and long-term objective are deeply detailed in Directive 2008/50/EC of the European 
102 impact on health (Schwartz et al., 1996; WHO, 2012, 2003; Liu and Peng, 2018; IARC, 2013;

103 Niranjan and Thakur, 2017; Janssen et al., 2011) they are of great interest for the project.

104 Within Polluscope, the purposes of this paper consist of:

-Selecting the sensors in accordance with the project expectations (measurement performance, size, price, etc.); and

-Assessing the selected sensors to determine precisely their ability to be used in the project.

\section{METHODS}

\subsection{Sensor selection protocol}

\section{$111 \quad$ 2.1.1 Pre-selection}

112 Among the main expected sensors specifications (listed in Table 1, measurement ranges were

113 set in accordance with the maximum measured hourly levels in Île-de-France by Airparif from 1141990 to $2017\left(\mathrm{O}_{3}: 170 \mathrm{ppb}, \mathrm{NO}_{2}: 270 \mathrm{ppb}\right.$ and $\left.\mathrm{PM}_{10}: 660 \mu \mathrm{g} \mathrm{m}^{-3}\right)$. BC hourly mean values up to 115 tens of micrograms per cubic meter were measured close to major traffic axes. As one of the 116 objectives of this work is to monitor the exposure in different environments (inside, outside, in a 117 car, etc.) throughout the day, a fine time resolution (below 5 min) was needed. Furthermore, some 118 practical requirements were added due to the need to have sensors able to measure all day long, 119 whatever the season, and which are easy to carry every day of the week. This is why a long 120 enough battery life was necessary, as well as being light weight and having a large working 121 temperature range. In addition to these fundamental specifications, the sensors had to present a 122 detection limit, a precision and an accuracy in accordance with the project goals. Moreover, close 
123 attention had to be paid to other possible issues, such as interference from other pollutants, long124 term drift, lack of data storage capacity, etc.

125 Tens of sensors measuring the pollutants of interest existed, but very few matched our 126 expectations. First, the sensor units must be commercially available; nevertheless, beta versions

127 of sensor units were considered in order to give a chance to almost commercialized units. 128 Furthermore, the considered units had to be built for mobile measurements.

129 According to these specifications, a pre-selection was made, mostly based on a bibliographic 130 survey. About fifty articles were studied; they are listed in Table S1 in the appendix.

131 At the end of the pre-selection stage, eight sensors fulfilling or mostly fulfilling the 132 requirements were chosen to be tested. Unfortunately, some devices gave aberrant values, this is 133 why no VOC results are presented in this work even if it was a pollutant of interest.

\section{$134 \quad$ 2.1.2 Testing strategy}

135 The first step consisted of testing the sensors in static measurements, for up to 15 days, in 136 ambient air, close to fixed reference instruments. These measurements took place at Site 137 instrumental de recherche par télédétection atmospherique (SIRTA $\left.{ }^{2}\right)$ which is an atmospheric 138 observatory belonging to the research insfrastructure ACTRIS ${ }^{3}$. For particulate matter, the 139 reference instruments were Fidas (Palas), TEOM 1405F for $\mathrm{PM}_{10}$ (Thermo Scientific), TEOM 1401400 for $\mathrm{PM}_{2.5}$ and $\mathrm{PM}_{1}$ (Thermo Electron) and Aethalometer AE33-7 for BC (Magee Scientific). 141 For gas monitoring, the reference instruments were T200UP for nitric oxides and T400 for ozone 142 (Teledyne). The time step was 1 minute for every instrument, except for TEOM, which was 15

\footnotetext{
${ }^{2} \mathrm{http}: / /$ sirta.ipsl.fr

${ }^{3}$ https://www.actris.eu/
} 
143 minutes. The reference instruments' inlets were situated on the roof of the building

144 (approximately 4 meters above ground level). Sensor units were fastened under a steel shelter

145 (next to the reference instruments building) in order to be protected from rain; they sampled the

146 air at around 2.5 meters above ground level. The units were powered all day long. This first step

147 consisting of static measurements compared with reference instruments aimed to be a first 148 assessment of sensor accuracy. For this kind of test, sensors are usually assessed compared with

149 reference instruments by using root mean squared error (RMSE) and Pearson correlation $R^{2}$.

150 However, these two indicators do not totally assess the capability of the sensor units to be used in

151 field campaigns. First, RMSE is too sensitive to brief discrepancies between the reference

152 instrument and the sensor. For instance, if two time series are very similar most of the time, but a

153 sudden and significant difference occurs, the RMSE could be large. The Pearson correlation is a 154 good indicator, but only for the linear domain. However, for extreme values, sensors could 155 measure outside their linear domain. Therefore, other correlation coefficients dealing with non156 linear domains are needed. This is why a tool developed by Fishbain et al. (2017) that 157 quantitatively assesses the sensors was used. The algorithm is called SET for sensor evaluation 158 toolbox, and as it is well described in the paper, only a brief explanation is given here. The SET 159 adds six parameters to the two above-mentioned (RMSE and Pearson correlation): the Kendall 160 and Spearman correlation (assessing the non-linear correlation), the presence (quantifying the 161 data loss), the source analysis (not used in the present work because not relevant in this context), 162 the match score (a metric assessing the ranking order similarities) and the lower frequencies 163 energy (LFE, estimating the sensor ability to properly capture the signal variability). Then, an 164 integrated performance index (IPI) is calculated from the eight parameters; this index goes from 0 165 to 1 and the higher the IPI, the better is the sensor. 
166 The second step consisted of mobility/reproducibility tests. These tests were done to assess the

167 ability of the sensors to measure not only in static positions but also in motion. To assess the 168 reproducibility of the measurements, a group of three persons were equipped, each with one unit

169 of the selected sensors. The measurements lasted one working day and took place by following a 170 route previously set, as done in scripted exposure studies (Jarjour et al., 2013). The itinerary was 171 chosen to pass through different environments (public bus, tramway, metro, office and restaurant) 172 in downtown and suburbs of Paris. The route also went close to Airparif monitoring stations 173 (www.airparif.fr), so as to get some "reference points" throughout the day. This itinerary is 174 plotted in Fig. 1.

\section{$175 \quad 2.2$ Selected sensors assessment}

176 Once the selection tests were over and the sensors were selected, it was of primary importance 177 to conduct a robust assessment of these instruments to be used in the field campaigns. The 178 different steps of this assessment are described below.

\section{$179 \quad$ 2.2.1 Reproducibility tests}

180 Fifteen sensor units were purchased for the "real" campaigns, all of them had to measure in the 181 same way, which is why reproducibility, accuracy and precision had to be properly checked. 182 From June $26^{\text {th }}$ to July $2^{\text {nd }} 2018$, all fifteen units of each selected sensor were tested 183 simultaneously in static measurements co-located with reference instruments just like during the 184 selection step.

\subsubsection{Controlled chamber tests}

186 The second step's goal was to assess the sensors' sensitivity to humidity, reactivity and 187 interferences to pollutant level changes as well as the sensors' signal to noise ratio under constant 188 conditions. These tests were conducted in a controlled chamber where the humidity is controlled 
189 (from approximately $40 \%$ to $80 \%$ relative humidity, the entire cycle lasted one hour, two cycles

190 were conducted) as well as $\mathrm{NO}_{2}$ concentration (from 0 to $200 \mathrm{ppb}$ ). These are the typical orders

191 of magnitude the sensors will have to deal with due to environmental changes during the coming

192 campaigns. $\mathrm{NO}_{2}$ variation is of course useful to characterize sensors measuring $\mathrm{NO}_{2}$, but this test

193 was also relevant for other gas sensors, as some of these devices can suffer from $\mathrm{NO}_{2}$

194 interference.

\section{$195 \quad$ 2.2.3 Feasibility campaign}

196 Finally, a feasibility campaign was performed, which consisted of a rehearsal or a practice for 197 the coming "real" campaigns. In other words, the selected sensor units were worn by 198 collaborators all day long for a week, in the exact same conditions that would occur during the 199 real campaigns. The goal here was to check for any issues of any kind (battery life, inlet 200 sampling, data flow, data processing, etc.) This exercise also allowed us to validate the final 201 campaign protocol.

\section{$202 \quad$ 2.2.4 Final sensor selection}

203 The authors have decided to anonymize the non-selected sensors in order not to discredit devices

204 that did not give good results during our experiments but which may have been improved since 205 then. The final selected sensors are presented in detail in this work: the AE51, the Cairclip and 206 the Canarin; below called, respectively, sensor A, sensor C and sensor F (technical details about 207 measurement principle are shown in appendix A).

\section{RESULTS}

209 Eight sensors were tested during the selection tests. The main specifications are listed in 210 Table 2.

\section{$211 \quad 3.1$ Static ambient air tests}


212 For static ambient air tests the results of six of them are presented (the sensors $\mathrm{G}$ and F showed

213 aberrant values). An example of the time series is given for the sensors A, B and C in Fig. 2 and

214 gives a preliminary assessment of the sensors' reliability. As shown in Fig. 2, the results from

215 several days of continuous measurements of sensors versus reference instruments are

216 heterogeneous among the different sensors and thus difficult to assess. The first time series

217 exhibits a BC sensor (black line), which gave results very close to the reference instrument (grey

218 line). For this sensor, the results were satisfying: the two lines are almost always overlapping.

219 This first basic tool (studying the time series) identified sensor A as being in agreement with the

220 project expectations.

221 However, the results were not always that unambiguous, and some sensors gave medium 222 results like the nitrogen dioxide sensors presented in the second time series (Fig. 2). For these two 223 devices, it is difficult to assess the performance of the sensors by only using the time series. 224 Furthermore, the difference with the reference instrument and the correlation are not the only 225 characteristics to focus on, but also the medium term shifting, the lack of data, dynamics, etc. are 226 important. This is why the SET tool (presented in the methods section) is relevant.

227 In Table 3, the integrated performance index (IPI) and the other SET results are presented for 228 the six sensors used in this work. The measurements time bases and the dates of the considered 229 period of time are also given here. BC measurements were done only by sensor A. The satisfying 230 performance of this sensor demonstrated with the time series is corroborated by the SET 231 evaluation, with a very good IPI of 0.91 , which is due to the high results for every single 232 parameter. Ozone was only measured by sensor B. For this pollutant, the IPI is mediocre with a 233 value of 0.46 . This is explained by the non-negligible data loss: the presence is 0.75 , which 234 means that one value out of four is missing. Furthermore, the RMSE is high (15 ppb) compared 235 with the mean value of $8 \mathrm{ppb}$, and even the match score $(0.3)$ is poor. Three sensors measured 
$236 \mathrm{NO}_{2}$. The best one was sensor B, with an IPI of 0.76 and a RMSE of $5 \mathrm{ppb}$. Sensor C gave poor

237 results and even aberrant values highlighted by very low correlation coefficients (below 0.15 ).

238 Sensor D has a fair correlation coefficient (higher than 0.5) but suffers from a large RMSE

239 (37 ppb), poor match score (0.24) and quite significant data loss (presence of $67 \%$ ). Particulate

240 matter was measured by three sensors: sensor $\mathrm{B}$ measured $\mathrm{PM}_{10}$ and the sensors $\mathrm{E}$ and $\mathrm{F}$

241 measured $\mathrm{PM}_{10}, \mathrm{PM}_{2.5}$ and $\mathrm{PM}_{1}$. Sensor F gave the best results for all the PM sizes, with an IPI of

$2420.64,0.80$ and 0.78 for $\mathrm{PM}_{10}, \mathrm{PM}_{2.5}$ and $\mathrm{PM}_{1}$, respectively. The others gave a much lower IPI.

243 The major advantage of sensor $\mathrm{F}$ is the data availability, which does not suffer from data loss. Its

244 match score is acceptable for the three PM sizes (always larger than 0.6) although this parameter

245 is lower than 0.43 for the others. Even if its RMSE is large, sensor $\mathrm{F}$ gives a relevant 246 approximation of the PM concentration.

247 This first static ambient test led us to rule out sensor E, which gave aberrant values for $\mathrm{PM}_{10}$, 248 as well as sensor D because of its non-satisfactory results. Sensor B was kept despite the 249 mediocre results for $\mathrm{O}_{3}$ (IPI of 0.46 ) and $\mathrm{PM}_{10}$ (IPI of 0.40), thanks to its multi-pollutant 250 measuring ability and because the producing firm should improve the sensor before the next 251 testing step. Sensor C gave here poor results, but the authors were aware that good results had 252 been obtained with this device, and it was suggested that these unsatisfactory results could be due 253 to an out-of-date electrochemical sensing cell or an inappropriate storage, which would lower the 254 sensor performance. This is why new units of this sensor were purchased for the following testing 255 steps.

\section{$256 \quad 3.2$ Mobility/reproducibility test}

257 This test involved the following sensors: sensor B, sensor C and sensor F. The BC sensor A 258 was not involved in this mobility tests because studies (Ezani et al., 2018; Lin et al., 2017) have 259 already demonstrated its ability to perform mobile measurements. Fig. 3 and Fig. 4 (and Fig. S1 
260 in Appendix) show, for the three units of each sensor, the entire time series for every sensor of 261 each pollutant and the comparison with the Airparif stations when the route goes by these stations.

262 Fig. 3 refers to the $\mathrm{NO}_{2}$ sensors. The whole-day time series shows that sensors $\mathrm{C}$ present a better

263 reproducibility between the units than sensors B. Secondly, the sensors C show a better dynamic 264 response whereas sensors B present averaged values. Moreover, this figure demonstrates the 265 sensitivity of the sensors $\mathrm{C}$ to monitor the environmental changes. Three specific environments 266 are pointed out in the time series: "Opéra" is an Airparif monitoring station classified as traffic 267 influenced, "Restaurant" refers to the lunch break, which took place in a cafeteria and "Bus" 268 stands for bus travel. Sensor C was able to identify different levels associated with different 269 environments: $\mathrm{NO}_{2}$ was high (around $50 \mathrm{ppb}$ ) during the time spent close to the Opéra traffic 270 location, low (about $10 \mathrm{ppb}$ ) in the restaurant (indoors) and presented strong variations during bus 271 travel. Furthermore, these sensors quickly detected the environment changes. On the contrary, 272 sensors B were very slow to monitor pollutant level variations, and were unable to properly 273 discriminate environmental changes. Below the main time series, graphs allow us to estimate the 274 accuracy of the sensors against Airparif stations, which can be considered as reference 275 measurements. Except for the "Paris centre" station, the three sensors $\mathrm{C}$ were in the right range; 276 the variations were also well monitored, especially for the "Célestins" and "Opéra" stations. The 277 sensors B never showed clear variation, and a significant difference existed between the three 278 units (up to tens of ppb for the "RN2" station). Fig. 4 shows the mobility test results for $\mathrm{PM}_{10}$ 279 sensors B and F. The time series depict poor consistency between the units, especially for sensor $280 \mathrm{~B}$, for which a gap of up to $50 \mu \mathrm{g} \mathrm{m}^{-3}$ was observed. The comparison with the Airparif stations 281 shows that sensors B gave poor results: for the stations "Paris centre" and "Bobigny", the sensor 282 values were significantly different from the reference; for the "Opéra" station, results were fair (a 283 shift of about $10 \mu \mathrm{g} \mathrm{m}^{-3}$ appears); and for the "RN2" station, the difference between the three 284 units was substantial (about $30 \mu \mathrm{g} \mathrm{m}^{-3}$ ). Results were better for the sensors F: the sensors were 
285 almost always in the range of the reference station, with an inter-sensor difference not larger than $28620 \mathrm{\mu g} \mathrm{m}^{-3}$. Nevertheless, sensors $\mathrm{F}$ results were poor for the "Bobigny" station, for which the 287 difference with the reference went up to $30 \mu \mathrm{g} \mathrm{m}^{-3}$. Three units of each sensor $(\mathrm{B}, \mathrm{C}$ and $\mathrm{F})$ were tested during this mobility steps. Sensors C and F presented the best results.

\subsection{Selected sensors}

290 The BC sensor A gave satisfying results and was therefore selected for the next step. The 291 sensors $\mathrm{C}$ gave suitable results during the mobility tests, which confirms the hypothesis that a 292 deteriorated unit was used during the static measurement tests. This sensor gave better results 293 than sensor $\mathrm{B}$ for $\mathrm{NO}_{2}$. The PM sensor $\mathrm{F}$ gave more accurate results than sensor $\mathrm{B}$. For this 294 reason and because of the poor results for $\mathrm{NO}_{2}$ and $\mathrm{O}_{3}$, sensor $\mathrm{B}$ was excluded.

295 The retained sensors were sensors $\mathrm{A}(\mathrm{BC}), \mathrm{C}\left(\mathrm{NO}_{2}\right)$ and $\mathrm{F}\left(\mathrm{PM}_{10}, \mathrm{PM}_{2.5}\right.$ and $\left.\mathrm{PM}_{1}\right)$. The next 296 section deals with the assessment of their capabilities. Several units of each of the three sensors 297 were purchased: 15 units for the sensors $\mathrm{C}$ and F, and 6 units for the sensor $\mathrm{A}$ (due to its high 298 cost).

3.4 Reproducibility tests in static measurements

300 An overview of the results is presented in Fig. 5. From the top to the bottom, are presented the $301 \mathrm{BC}, \mathrm{NO}_{2}$ and $\mathrm{PM}_{2.5}$ measurements. The reference instrument is plotted in black and the sensors in 302 coloured lines. Generally, the sensors closely followed the reference instrument trend even if 303 some discrepancies were observed. The BC sensors A were very accurate, despite some noisy 304 periods. On the whole, the $\mathrm{NO}_{2}$ sensors $\mathrm{C}$ overestimated the concentration, this is certainly due to 305 the low $\mathrm{NO}_{2}$ ambient concentration compared with the limit of detection (20 ppb according to the 306 manufacturer's specifications). This behaviour was already observed by Duvall et al. (2016).

307 Even if these sensors were still able to monitor the global variability in these conditions, another 308 measurement campaign was conducted. The objective was to submit the sensors to $\mathrm{NO}_{2}$ ambient 
309 levels higher than the devices' detection limit. From August 28th to September 4th, fifteen $\mathrm{NO}_{2}$

310 sensors $\mathrm{C}$ were used to measure conditions at the Airparif station close to a major road (Paris ring

311 road). The results overview is presented in Appendix (Fig. S2), the rest of this work is based on

312 the results from this experiment. Concerning the PM sensors F, they were both quite close to the

313 reference and very reproducible to one another, except for one unit plotted in light green, which

314 presented erratic values. For the $\mathrm{PM}_{2.5}$, the mean RMSE is $6 \mu \mathrm{g} \mathrm{m}^{-3}$, which is fairly low

315 compared with the measured concentration in mobility in Paris and its suburbs (often higher than $\left.31640 \mu \mathrm{g} \mathrm{m}^{-3}\right)$.

317 The SET results for the BC sensors are presented in details in Table S2 in appendix. The IPI is 318 high (around 0.8) for all the sensor units. These sensors did not suffer from data loss at all (the 319 presence parameter is always almost $100 \%$ ). The reproducibility between the units can be 320 quantified by the measuring range, defined as the average of the difference across the units 321 between the maximum and the minimum for each measurement date. For these BC sensor units, 322 the measurement range is $616 \mathrm{ng} \mathrm{m}^{-3}$, which is not negligible but below the mean concentration 323 value.

324 The SET results for the fifteen $\mathrm{NO}_{2}$ sensor units (Table $\mathrm{S} 3$ in appendix) are homogeneous, the 325 IPI spans from 0.75 to 0.79 . The mean measured concentration was above $40 \mathrm{ppb}$, which is 326 higher than the limit of detection. This leads to good correlation coefficients (above 0.76 for the 327 mean Pearson coefficient) and reasonable RMSE compared with the ambient levels (mean RMSE 328 is $14 \mathrm{ppb}$ ). Moreover, data loss was very uncommon as shown by the presence parameter.

329 The PM SET results are presented in Tables S4, S5 and S6 in appendix for the three PM sizes. 330 Regarding $\mathrm{PM}_{2.5}$ sensor units, the IPI is above 0.68 , except for the sensor unit F7, which was 331 defective (plotted in light blue in Fig. 5). The presence is higher than 0.72 (except for F6 at 0.62): 332 the sensor units F data loss was low. The correlation coefficients were never below 0.6, which 
333 shows good agreement with the reference. The mean measuring ranges for the three classes of

$334 \mathrm{PM}$ are $16 \mu \mathrm{g} \mathrm{m}^{-3}, 19 \mu \mathrm{g} \mathrm{m}^{-3}$ and $20 \mu \mathrm{g} \mathrm{m}^{-3}$ for $\mathrm{PM}_{1}, \mathrm{PM}_{2.5}$ and $\mathrm{PM}_{10}$, respectively. These 335 measuring ranges are not negligible but the mean RMSE values are lower: $4 \mu \mathrm{g} \mathrm{m}^{-3}, 6 \mu \mathrm{g} \mathrm{m}^{-3}$ 336 and $14 \mu \mathrm{g} \mathrm{m}^{-3}$ for $\mathrm{PM}_{1}, \mathrm{PM}_{2.5}$ and $\mathrm{PM}_{10}$, respectively. This means that a discrepancy existed 337 between the units, but the general agreement with the true value is acceptable.

\subsection{Chamber controlled test}

339 Fig. 6 shows the results of this test. The top chart represents the controlled parameters 340 monitored: humidity and $\mathrm{NO}_{2}$ concentration. The three others are the sensors results.

341 The sensor F values were always zero: it was not affected at all by humidity changes (at least

342 in the absence of PM). The BC sensor A was clearly affected by humidity variations as it showed

343 BC concentration variations at the same time as humidity ones. When the humidity was 344 decreasing, a positive artefact of up to $250 \mathrm{ng} \mathrm{m}^{-3}$ was observed. Conversely, a negative artefact 345 of $150 \mathrm{ng} \mathrm{m}^{-3}$ was reached when the humidity decreased to $40 \% \mathrm{RH}$. The mean value and the 346 standard deviation of this sensor over the constant humidity period were respectively $15 \mathrm{ng} \mathrm{m}^{-3}$ 347 and $14 \mathrm{ng} \mathrm{m}^{-3}$, which is low compared with ambient levels. Two $\mathrm{NO}_{2}$ sensor units $\mathrm{C}$ were 348 measuring in the chamber. They presented a sensitivity to humidity changes with the same 349 pattern as the BC sensor. The positive artefacts went up to $66 \mathrm{ppb}$ and the negative artefact was 350 constrained at zero (there is no negative value). We have also to note that the two sensor units 351 gave results close to one another, with a RMSE of 7 ppb. Over constant humidity and a $\mathrm{NO}_{2}$ 352 concentration of $25 \mathrm{ppb}$, their mean values were $21 \mathrm{ppb}$ and $26 \mathrm{ppb}$ with a standard deviation 353 around $1 \mathrm{ppb}$.

354 The second test conducted in the controlled chamber was the $\mathrm{NO}_{2}$ variation (Fig. S3 in 355 Appendix). This experiment consisted of a succession of one-hour steps of $0 \mathrm{ppb}, 50 \mathrm{ppb}$, 
$356100 \mathrm{ppb}, 200 \mathrm{ppb}, 0 \mathrm{ppb}$ and a final longer $50 \mathrm{ppb}$ stage over several hours. Inside the chamber, 357 the relative humidity was set at $60 \%$ and there is no particulate matter.

358 The ability of the sensors to monitor quick concentration changes was demonstrated here as the 359 two sensor units reacted at the same time as the reference instrument. The sensor units were able 360 to monitor increases (up to $200 \mathrm{ppb}$ ) and decreases down to $0 \mathrm{ppb}$. However, a gap can be 361 observed between the sensor units and the reference, the associated RMSE is $11 \mathrm{ppb}$ and $15 \mathrm{ppb}$. 362 For the final longer step at $50 \mathrm{ppb}$, the RMSE stood at $9 \mathrm{ppb}$ and $2 \mathrm{ppb}$.

363 To conclude for the controlled chamber tests, although sensor F presented no artefacts due to 364 humidity changes (with a zero concentration of PM), the sensors $\mathrm{A}$ and $\mathrm{C}$ were sensitive to 365 humidity. During the following campaigns, this will have to be taken into account, a correction or 366 an invalidation protocol may be needed.

\section{$367 \quad 3.6$ Feasibility campaign}

368 The feasibility campaign was conducted with fifteen volunteers from Monday 18th to Friday 369 22nd June 2018. One sensor unit $\mathrm{C}$ and one sensor unit $\mathrm{F}$ were given to each volunteer and six 370 sensor units A were shared between the participants. This campaign was conceived as a proof of 371 the Polluscope concept, thus, only a limited analysis of the results was done.

372 Globally, the campaign was a success: all the sensor units were worn for the whole week. The 373 data availability (the time resolution was one minute) is $66 \%$ for sensors A and $69 \%$ for sensors 374 F, which can be considered as a satisfying result. The data loss was due to minor problems and 375 routine maintenance (filter change, turning on and off, powering, etc.). However, the data 376 availability for the sensors $\mathrm{C}$ only reaches $41 \%$. This was caused by storage memory erasure 377 when the sensor ran out of power. The coming campaigns protocols will prevent this issue.

378 Generally, the data availability was slightly lower than during the previous tests, this was due to 
the campaign environment and the fact that the sensor units were operated by volunteers without 380 expert skills.

381 Fig. 7 shows the results of the three sensor units worn for the whole week by a volunteer. Four 382 kinds of environments are pointed out: "indoor" for the time spent inside, "polluted indoor" for 383 emitting activities conducted indoors (cafeteria, smoking or cooking, for instance), "commuting" 384 journeys (whatever the travel mode) and "outdoor" for the time spent outside any building. The 385 indoor environment is the more frequent environment, nevertheless, the spikes were usually 386 observed during commuting or in polluted indoor environments. The major $\mathrm{BC}$ and $\mathrm{NO}_{2}$ peaks 387 occurred most frequently during commuting trips. Inversely, the highest particulate values were 388 measured during "polluted indoor" episodes. An example of contrasted environments 389 (commuting, indoors and tobacco smoke in indoor environment) measurements is presented in 390 Appendix, Fig. S4. During the campaign, artefacts due to quick environmental change (studied in 391 the controlled chamber in Section 3.5) were observed; this is more detailed in Appendix, Fig. S5).

392 This feasibility campaign demonstrated the capability of the Polluscope protocol to conduct a 393 campaign lasting a whole week with volunteers. The results from the sensors enable us to 394 discriminate several emitting activities; a preliminary estimation of the personal exposure is thus 395 available.

\section{DISCUSSION}

397 The first stages of Polluscope (the selection and assessment of the sensors) have been 398 conducted. The AE51 (BC), the Cairclip $\left(\mathrm{NO}_{2}\right)$ and the Canarin $\left(\mathrm{PM}_{10}, \mathrm{PM}_{2.5}\right.$ and $\left.\mathrm{PM}_{1}\right)$ have 399 been selected and assessed. The feasibility campaign demonstrated that these three sensors are 400 reliable enough to be used for full-scale campaigns involving volunteers from the general public. 401 Their ability to discriminate different environments (commuting trips, polluted or clean indoor 402 environment, etc.) has been proven. 
403 For the static measurement assessments, we used the SET algorithm designed by Fishbain et al. 404 (2017), available as an open source resource ${ }^{4}$. In their article, they presented results from 25 405 AQMesh $\mathrm{NO}_{2}$ sensor units that had taken measurements for about three months in static positions. 406 The mean related IPI is 0.58 , this is very close to our mean Cairclip IPI (from the reproducibility 407 test) of 0.54 ; even if the Cairclip is designed for mobile measurements whereas the AQMesh 408 system is designed for static monitoring (i.e. expecting to have a better performance than a 409 portable device). Knowing the successful deployment of the AQMesh sensor units, this result 410 demonstrates the reliability of the Cairclip. Fishbain et al. (2017) also used the SET with PM 411 sensors (DC1700 Dylos and GeoTech), the mean resulting IPI is 0.63. The PM 2.5 Canarin sensor 412 used in our study gave significantly better results with a mean IPI of 0.73 .

413 The SET algorithm was also used by Broday and the Citi-Sense Project Collaborators (2017).

414 They presented unpublished results from about three months of ambient air measurements of six $415 \mathrm{PM}_{10}$ sensor units located in Ostrava, Czech Republic. The mean IPI is 0.72 , which is very close 416 to the result from our study (0.73).

417 Due to its recent release, SET algorithm results have not been published in other articles yet. 418 To the best of our knowledge, our study is the first to apply the SET evaluation to the AE51 and 419 the Cairclip sensors. However, these two devices have been largely used and several results have 420 been published, some of the more relevant for our study are discussed below.

\section{$421 \quad 4.1$ Static comparison}

422 Lin et al. (2017) compared AE51 with reference instruments and found a good mean 423 correlation of 0.77 . Viana et al. (2015) conducted a study involving six AE51 and a reference

\footnotetext{
${ }^{4}$ fishbain.net.technion.ac.il
} 
424 station in static measurements, the correlation coefficient was above 0.75 . In our study, the mean 425 correlation coefficient was 0.80 . This higher agreement may be due to higher inlet flow 426 (150 $\mathrm{mL} \mathrm{min}^{-1}$ compared with $100 \mathrm{~mL} \mathrm{~min}^{-1}$ in the Viana et al. (2015) study) or coarser time 427 resolution (1 min in our study and $1 \mathrm{sec}$ in the Lin et al. (2017) study).

428 Several other studies have pointed to the good results of this black carbon sensor in agreement 429 with our results (Cai et al., 2014; Gillespie et al., 2017; Velasco and Tan, 2016).

430 The recent low-cost sensors review by the World Metrological Organization (Lewis et al., 431 2018) described several performance evaluation programs as the work supported by the United 432 States Environment Protection Agency (EPA) ${ }^{5}$; they described an air sensor toolbox where the 433 main performances of tens of sensors were gathered and compared. For the Cairclip $\mathrm{NO}_{2}$ sensor, 434 the EPA and Jiao et al. (2016) state that a correlation coefficient between 0.42 and 0.76 was 435 obtained with reference instruments. Our mean Pearson correlation was 0.76, which is in the high 436 part of the EPA range. The additional information given by the SET algorithm in our study is the 437 good match score of 0.64 and the absence of data loss (the presence parameter reaches almost $438100 \%$ ). Another example is the study conducted by Spinelle et al. (2015). They found a 439 correlation of up to 0.75 for the Cairclip sensor units. This result is both in the EPA range and 440 close to our result of 0.76 .

441 Due to its new release, only a few research works including Canarin have been conducted. For 442 instance, Tse et al., 2018B presented a project based on static measurements from four Canarin 443 units. Some tests were conducted in Bologna, Italia, and $\mathrm{PM}_{10}$ maps have been produced. These 444 works were preliminary and the most accomplished article about Canarin sensor is certainly the 445 one conducted by Tse et al., 2018A. They deployed nine Canarin units in a library inducted at the

\footnotetext{
${ }^{5}$ https://www.epa.gov/air-sensor-toolbox
} 
446 UNESCO world heritage list. The sensors were measuring 24/7 for months (from Summer 2017

447 to Spring 2018), which enabled to show that a clear diurnal pattern occurred with higher levels 448 during night time. On a longer period of time it was the winter season which experienced more 449 pollution. This protocol also permitted to quantify that $56 \%$ of the time, the $\mathrm{PM}_{2.5}$ air pollution 450 level was low according to the EPA standards (below $12 \mu \mathrm{g} \mathrm{m}^{-3}$ ). The coming improvements 451 announced in these three articles suggested a wider use of the Canarin in a near future. Overall, 452 these works underlined the promising capabilities of this sensor. The present paper confirmed this 453 first evaluation and went a step further (larger amount of units, mobile measurements, etc.) to 454 prove the ability to use the Canarin to equip volunteers for the personal exposure quantification.

\subsection{Mobile measurements}

456 It is usually more difficult to robustly assess sensor accuracy in mobile measurements as the 457 reference instruments are unlikely to be usable in motion. A metric that can be used is the 458 agreement between several units of portable sensors (previously assessed - or not - in static 459 measurements versus a reference instrument). This provides information on the reproducibility 460 and thus on the reliability of the mobile device. Another possibility is to compare the sensor 461 measurements with static stations considered as a reference if the mobility route goes close to this 462 kind of monitoring site.

463 The Ezani et al. (2018) study was based on mobile measurements performed with two AE51 464 units. There were no reference instruments but the correlation between the two units was good: 465 0.92. Lin et al. (2017) performed mobile AE51 measurements. Comparison was possible thanks 466 to 17 transient immobile periods (of less than one hour) nearby reference stations. The AE51 unit 467 showed an interquartile range agreement with the reference instrument of $82 \%$. High-resolution 468 mapping is possible with the AE51, as in the study of den Bossche et al. (2015) where sensor units were mounted on bikes in Antwerp, Belgium. A 50-meter resolution was obtained with an 
470 uncertainty of $25 \%$. Pant et al. (2017) performed a study aiming at quantifying personal 471 exposure to $\mathrm{BC}$ in New Delhi, India. The AE51 were given to volunteers and environments 472 (commuting, cooking, etc.) were distinguished.

473 Few studies have been published on the Cairclip sensor being used in mobile measurements, 474 especially compared with the abundant literature related to the AE51. The recent work by 475 Chambers et al. (2018) found no consistent relationship between $\mathrm{NO}_{2}$ concentrations and health 476 parameters. The authors state that the Cairclip was able to appropriately monitor personal 477 exposure and a clear diurnal cycle was observed but no more validation data was provided. The 478 study by Reid (2015) is based on the qualification of Cairclip sensors. Mobile measurements 479 were conducted with two Cairclip units in different environments: public transport, outdoor and 480 indoor. The sensors monitored interesting variability, especially close to traffic.

481 Lastly, Aguiari et al., 2018 introduced a possible use of the Canarin by attaching them to bikes.

\subsection{Methodology discussion}

483 Our work has revealed that the three selected sensors are appropriate for personal exposure assessment. Beyond that first result, the Polluscope selection and assessment methodology was also an outcome of this study.

486 It is now well known, even in the emerging field of small air quality sensors that a complete 487 sensor assessment is of primary importance to obtain reliable data. Some studies were only based 488 on laboratory experiments (Manikonda et al., 2016; $\mathrm{Ng}$ et al., 2018), but in-the-field calibration 489 was identified as necessary to properly assess the sensors' capabilities (Castell et al., 2017; 490 Schneider et al., 2017) as the results can be substantially different from laboratory-controlled 491 environments. For instance, during our tests, the Cairclip showed better results during the 492 controlled chamber tests. The ambient air tests were very useful to reveal that the Cairclip had 493 difficulties in measuring low ambient concentrations. 
494 As seen in the section concerning the reproducibility tests, non-negligible differences were 495 observed between units of the same sensor. This highlights the importance of testing several units 496 at a time. In this study, we conducted tests with 6 AE51, 15 Cairclip and 15 Canarin. For some of 497 the previously published studies, the small number of tested units was a limitation, for example, 498 Lin et al. (2017) (two AE51 and only one for the mobility tests), Ezani et al. (2018) (two AE51), 499 Duvall et al. (2016) (two Cairclip).

\section{$500 \quad 4.4$ Conclusions}

$501 \quad$ No remote sensor is perfect, and the three selected ones are the result of compromises and each 502 have strengths and weaknesses. The AE51 is accurate, its IPI (above 0.8) is higher than all other 503 sensors. This BC sensor is also reliable and easy to use with very little data loss. But it is 504 sensitive to humidity, which leads to some artefacts when quick environmental changes occur, 505 and its high price is also a weakness because fewer units can be purchased. The Cairclip is very 506 light and thus easy to carry all day long, but the storage memory is erased if the sensor unit runs 507 out of battery, thus it is consequently more demanding for the operator. Moreover, even if these 508 sensors demonstrated their ability to perform reliable measurements in mobile measurement (see 509 related section), the detection limit (20 ppb) is not appropriate for low $\mathrm{NO}_{2}$ levels. The Canarin is 510 able to send data via Wi-Fi and has a high storage capacity (several weeks of measurements), 511 which is useful when the data sending is not possible. Its robustness in mobility is also an 512 important advantage: the Canarin was the sensor that presented the highest data availability 513 during the feasibility campaign (69\%). This sensor showed satisfying results for the $\mathrm{PM}_{2.5}$ 514 measurements (IPI of 0.7) but substantially lower for $\mathrm{PM}_{10}$ (IPI of 0.4 ). Finally, the weight of the 515 sensor unit (it is quite heavy) is a drawback. 
516 Even if the three selected sensors have some weaknesses, their ability to be used in mobile 517 measurements has been demonstrated. For the coming campaigns, attention will be given to their 518 drawbacks.

519 To conclude, the Polluscope project is one of the few studies that has conducted an in-depth 520 sensor assessment including the most important following steps:

- Several kinds of tests were performed. Ambient air static measurement tests against reference instruments was the first assessment of the sensors in real atmosphere with natural meteorological parameters variability (temperature, humidity, wind speed and direction, etc.) The laboratory tests were of primary importance to quantify the sensors; responses to rapid atmospheric changes (i.e., humidity, pollutants levels). Mobile measurements were necessary as the project goal is to use the sensors for personal exposure, to be worn by volunteers.

-A large number of units of each sensor was tested to quantify the reproducibility and to eliminate problems arising from a single deficient unit.

-A robust multi-metric static measurement assessment with the SET algorithm was conducted in order to be as rigorous as possible in the assessment.

532 The next stage of the Polluscope project will be the full-scale campaigns involving fifteen 533 volunteers each week during the six weeks per studied season. These campaigns will take place 534 over two years and will involve 160 people. Even if the size of the project is already consistent, a 535 valuable perspective would be to recruit more volunteers over a larger area in order to increase 536 the representativeness of the study. 


\section{ACKNOWLEDGEMENTS}

540 This project was funded by the French agency: Agence nationale de la recherche (ANR -

541 http://www.agencenationale-recherche.fr/Projet-ANR-15-CE22-0018). Part of the equipment was

542 funded by iDEX Paris-Saclay, in the framework of the project ACE-ICSEN. Measurements from

543 the SIRTA station were performed within the ACTRIS research infrastructure under the H2020

544 grant agreement 654109. Additional support from CEA and CNRS are acknowledged. 


\section{REFERENCES}

548 Adgate, J.L., Church, T.R., Ryan, A.D., Ramachandran, G., Fredrickson, A.L., Stock, T.H., 549 Morandi, M.T. and Sexton, K. (2004). Outdoor, indoor, and personal exposure to VOCs in $550 \quad$ children. Environ. Health Perspect. 112(14): 1386-1392.

551 Aguiari, D., Delnevo, G., Monti, L., Ghini, V., Mirri, S., Salomoni, P., Pau, G., Im, M., Tse, R., 552 Ekpanyapong, M., and Battistini, R. (2018). Canarin ii : Designing a smart e-bike eco-system. 15th IEEE Annual Consumer Communications \& Networking Conference (CCNC) 1-6

554 Airparif - La qualité de l'air dans les enceintes du métro ou du rer, 555 https://www.airparif.asso.fr/pollution/air-interieur-metro, Accessed: 22 November 2018.

556 Airparif (2017). Bilan de la qualité de l'air 2016 - surveillance et information en Île-de-France.

557 Airparif (2018). Bilan de la qualité de l'air 2017 - surveillance et information en Île-de-France.

558 Airparif (2019). Bilan de la qualité de l'air 2018 - surveillance et information en Île-de-France.

559 Borghi, F., Spinazzè, A., Rovelli, S., Campagnolo, D., Del Buono, L., Cattaneo, A., and Cavallo, 560 D.M. (2017). Miniaturized monitors for assessment of exposure to air pollutants: A review. Int. $561 \quad$ J. Environ. Res. Public Health 14(8): 909.

562 den Bossche, J.V., Peters, J., Verwaeren, J., Botteldooren, D., Theunis, J. and Baets, B.D. (2015). 563 Mobile monitoring for mapping spatial variation in urban air quality: Development and validation of a methodology based on an extensive dataset. Atmos. Environ. 105: 148-161.

565 Broday, D.M. and the Citi-Sense Project Collaborators (2017). Wireless distributed 566 environmental sensor networks for air pollution measurement: the promise and the current 567 reality. Sensors 17(10): 2263.

568 Burkart, J., Steiner, G., Reischl, G., Moshammer, H., Neuberger, M. and Hitzenberger, R. (2010). 
569 Characterizing the performance of two optical particle counters (grimm opc1.108 and opc1.109) $570 \quad$ under urban aerosol conditions. J. Aerosol Sci. 41(10): 953-962.

571 Cai, J., Yan, B., Ross, J.M., Zhang, D., Kinney, P.L., Perzanowski, M.S., Jung, K., Miller, R.L. 572 and Chillrud, S.N. (2014). Validation of microaeth ${ }^{\circledR}$ as a black carbon monitor for fixed-site 573 measurement and optimization for personal exposure characterization. Aerosol Air Qual. Res. $574 \quad 14(1): 1-9$.

575 Castell, N., Dauge, F.R., Schneider, P., Vogt, M., Lerner, U., Fishbain, B., Broday, D. and 576 Bartonova, A. (2017). Can commercial low-cost sensor platforms contribute to air quality 577 monitoring and exposure estimates? Environ. Int. 99: 293-302.

578 Castell, N., Kobernus, M., Liu, H.-Y., Schneider, P., Lahoz, W., Berre, A.J. and Noll, J. (2015). 579 Mobile technologies and services for environmental monitoring: The Citi-sense-MOB $580 \quad$ approach. Urban Clim. 14(Part 3): 370-382.

581 Chambers, L., Finch, J., Edwards, K., Jeanjean, A., Leigh, R. and Gonem, S. (2018). Effects of 582 personal air pollution exposure on asthma symptoms, lung function and airway inflammation. 583 Clin. Exp. Allergy, 48(7): 798-805.

584 Deville Cavellin, L., Weichenthal, S., Tack, R., Ragettli, M.S., Smargiassi, A. and Hatzopoulou, 585 M. (2016). Investigating the use of portable air pollution sensors to capture the spatial 586 variability of traffic-related air pollution. Environ. Sci. Technol. 50(1): 313-320.

587 Duvall, R.M., Long, R.W., Beaver, M.R., Kronmiller, K.G., Wheeler, M.L. and Szykman, J.J. 588 (2016). Performance evaluation and community application of low-cost sensors for ozone and $589 \quad$ nitrogen dioxide. Sensors 16(10): 1698.

590 European Environment Agency (2017). Air quality in Europe 2017 report. European $591 \quad$ Environment Agency. 
592 Ezani, E., Masey, N., Gillespie, J., Beattie, T.K., Shipton, Z.K. and Beverland, I.J. (2018).

593 Measurement of diesel combustion-related air pollution downwind of an experimental 594 unconventional natural gas operations site. Atmos. Environ. 189: 30-40.

595 Fishbain, B., Lerner, U., Castell, N., Cole-Hunter, T., Popoola, O., Broday, D.M., Iñiguez, T.M., 596 Nieuwenhuijsen, M., Jovasevic-Stojanovic, M., Topalovic, D., Jones, R.L., Galea, K.S., Etzion, 597 Y., Kizel, F., Golumbic, Y.N., Baram-Tsabari, A., Yacobi, T., Drahler, D., Robinson, J.A., 598 Kocman, D., Horvat, M., Svecova, V., Arpaci, A. and Bartonova, A. (2017). An evaluation 599 tool kit of air quality micro-sensing units. Sci. Total Environ. 575: 639-648.

600 Gao, M., Cao, J. and Seto, E. (2015). A distributed network of low-cost continuous reading 601 sensors to measure spatiotemporal variations of $\mathrm{PM}_{2.5}$ in Xi'an, China. Environ. Pollut. 199: $602 \quad 56-65$.

603 Gillespie, J., Masey, N., Heal, M.R., Hamilton, S. and Beverland, I.J. (2017). Estimation of 604 spatial patterns of urban air pollution over a four-week period from repeated 5-min 605 measurements. Atmos. Environ. 150: 295-302.

606 Hasenfratz, D., Saukh, O., Walser, C., Hueglin, C., Fierz, M., Arn, T., Beutel, J. and Thiele, L. 607 (2015). Deriving high-resolution urban air pollution maps using mobile sensor nodes. 608 Pervasive Mob. Comput. 16(Part B): 268-285.

609 Hinds, W.C. and Bellin, P. (1988). Effect of facial-seal leaks on protection provided by half-mask 610 respirator. Appl. Ind. Hyg. 3(5): 158-164.

611 Holstius, D.M., Pillarisetti, A., Smith, K.R. and Seto, E. (2014). Field calibrations of a low-cost 612 aerosol sensor at a regulatory monitoring site in California. Atmos. Meas. Tech. 7(4): 1121$613 \quad 1131$.

614 Hu, K., Sivaraman, V., Luxan, B.G. and Rahman, A. (2016). Design and evaluation of a 
616 Hu, K., Wang, Y., Rahman, A. and Sivaraman, V. (2014). Personalising pollution exposure 617 estimates using wearable activity sensors. In 2014 IEEE Ninth International Conference on 618 Intelligent Sensors, Sensor Networks and Information Processing (ISSNIP) (pp. 1-6).

619 IARC (2013). Air pollution and cancer. IARC Scientific Publications.

620 Janssen, N.A., Hoek, G., Simic-Lawson, M., Fischer, P., van Bree, L., ten Brink, H., Keuken, M., 621 Atkinson, R.W., Anderson, H.R., Brunekreef, B. and Cassee, F.R. (2011). Black carbon as an 622 additional indicator of the adverse health effects of airborne particles compared with $\mathrm{PM}_{10}$ and $623 \quad \mathrm{PM}_{2.5}$. Environ. Health Perspect. 119(12): 1691-1699.

624 Jarjour, S., Jerrett, M., Westerdahl, D., de Nazelle, A., Hanning, C., Daly, L., Lipsitt, J. and 625 Balmes, J. (2013). Cyclist route choice, traffic-related air pollution, and lung function: a $626 \quad$ scripted exposure study. Environmental Health 12(1): 14.

627 Jiao, W., Hagler, G., Williams, R., Sharpe, R., Brown, R., Garver, D., Judge, R., Caudill, M., 628 Rickard, J., Davis, M., Weinstock, L., Zimmer-Dauphinee, S. and Buckley, K. (2016). 629 Community air sensor network (Cairsense) project: evaluation of low-cost sensor performance 630 in a suburban environment in the south-eastern United States. Atmos. Meas. Tech. 9(11): 5281$631 \quad 5292$.

632 Klepeis, N.E., Nelson, W.C., Ott, W.R., Robinson, J.P., Tsang, A.M., Switzer, P., Behar, J.V., 633 Hern, S.C. and Engelmann, W.H. (2001). The national human activity pattern survey (NHAPS): 634 A resource for assessing exposure to environmental pollutants. J. Exposure Anal. Environ. $635 \quad$ Epidemiol. 11(3): 231-252.

636 Lewis, A.C., von Schneidemesser, E. and Peltier, R.E. (2018). Low-cost sensors for the 637 measurement of atmospheric composition: overview of topic and future applications. World 
639 Lin, C., Gillespie, J., Schuder, M., Duberstein, W., Beverland, I. and Heal, M. (2015). Evaluation 640 and calibration of aeroqual series 500 portable gas sensors for accurate measurement of 641 ambient ozone and nitrogen dioxide. Atmos. Environ. 100: 111-116.

642 Lin, C., Masey, N., Wu, H., Jackson, M., Carruthers, D.J., Reis, S., Doherty, R.M., Beverland, I.J. 643 and Heal, M.R. (2017). Practical field calibration of portable monitors for mobile 644 measurements of multiple air pollutants. Atmosphere 8(12): 231.

645 Liu, J.C. and Peng, R.D. (2018). Health effect of mixtures of ozone, nitrogen dioxide, and fine 646 particulates in 85 US counties. Air Qual. Atmos. Health. 11(3): 311-332.

647 Manikonda, A., Zikova, N., Hopke, P.K. and Ferro, A.R. (2016). Laboratory assessment of low$648 \quad$ cost PM monitors. J. Aerosol Sci. 102: 29-40.

649 Mead, M., Popoola, O., Stewart, G., Landsho, P., Calleja, M., Hayes, M., Baldovi, J., McLeod, 650 M., Hodgson, T., Dicks, J., Lewis, A., Cohen, J., Baron, R., Saffell, J. and Jones, R. (2013). 651 The use of electrochemical sensors for monitoring urban air quality in low-cost, high-density 652 networks. Atmos. Environ. 70: 186-203.

653 Ng, C.-L., Kai, F.-M., Tee, M.-H., Tan, N. and Hemond, H.F. (2018). A prototype sensor for in 654 situ sensing of fine particulate matter and volatile organic compounds. Sensors 18(1): 265.

655 Niranjan, R. and Thakur, A.K. (2017). The toxicological mechanisms of environmental soot 656 (black carbon) and carbon black: focus on oxidative stress and inflammatory pathways. Front. $657 \quad$ Immunol. 8: 763.

658 Pant, P., Habib, G., Marshall, J.D. and Peltier, R.E. (2017). PM 2.5 exposure in highly polluted 659 cities: a case study from New Delhi, India. Environ. Res. 156: 167-174.

660 Peng, I.H., Chu, Y.Y., Kong, C.Y. and Su, Y.S. (2013). Implementation of indoor VOC air 
Reid, B. (2015). A quantitative analysis of the Cairclip $\mathrm{O}_{3} / \mathrm{NO}_{2}$ sensor. Thesis, University of 664 Alberta.

665 Sante Publique France (2016). Impacts sanitaires de la pollution de l'air en France : nouvelles 666 données et perspectives. Sante Publique France.

667 Schneider, P., Castell, N., Vogt, M., Dauge, F.R., Lahoz, W.A. and Bartonova, A. (2017). 668 Mapping urban air quality in near real-time using observations from low-cost sensors and 669 model information. Environ. Int. 106: 234-247.

670 Schwartz, J., Dockery, D.W. and Neas, L.M. (1996). Is daily mortality associated specifically 671 with fine particles? J. Air Waste Manage. Assoc. 46(10): 927-939.

672 Sousan, S., Koehler, K., Thomas, G., Park, J.H., Hillman, M., Halterman, A. and Peters, T.M. 673 (2016). Inter-comparison of low-cost sensors for measuring the mass concentration of 674 occupational aerosols. Aerosol Sci. Technol. 50(5): 462-473.

675 Spinelle, L., Gerboles, M., Kok, G., Persijn, S. and Sauerwald, T. (2017). Review of portable and 676 low-cost sensors for the ambient air monitoring of benzene and other volatile organic 677 compounds. Sensors 17(7): 1520.

678 Tse, R., Aguiari, D., Chou, K.S., Tang, S.K., Giusto, D., and Pau, G. (2018A) Monitoring cultural 679 heritage buildings via low-cost edge computing/sensing platforms: The biblioteca joanina de 680 coimbra case study. 4th International Conference on Smart Objects and Technologies for $681 \quad$ Social Good, Goodtechs 148-152.

682 Tse, R., Monti, L., Prandi, C., Aguiari, D., Pau, G., and Salomoni, P. On assessing the accuracy 683 of air pollution models exploiting a strategic sensors deployment (2018B). 4th EAI 

58.

686 Velasco, A., Ferrero, R., Gandino, F., Montrucchio, B. and Rebaudengo, M. (2016). A mobile 687 and low-cost system for environmental monitoring: a case study. Sensors 16: 710.

688 Velasco, E. and Tan, S.H. (2016). Particles exposure while sitting at bus stops of hot and humid 689 Singapore. Atmos. Environ. 142: 251-263.

690 Viana, M., Rivas, I., Reche, C., Fonseca, A., Perez, N., Querol, X., Alastuey, A., Alvarez 691 Pedrerol, M. and Sunyer, J. (2015). Field comparison of portable and stationary instruments for 692 outdoor urban air exposure assessments. Atmos. Environ. 123(Part A): 220-228.

693 World Health Organization (2014). Seven million premature deaths annually linked to air 694 pollution. http://www.who.int/mediacentre/news/releases/2014/air-pollution/en/. Accessed: 22 $695 \quad$ November 2018.

696 World Health Organization (2012). Health effects of black carbon. World Health Organization.

697 World Health Organization (2003). Health aspects of air pollution with particulate matter, ozone 698 and nitrogen dioxide. World Health Organization, Regional Office for Europe. 
2 Table 1. Tested sensor specifications.

3 Table 2. Main expected specifications of the sensors.

4 Table 3. SET results for every sensor, the units are "ppb" for gases, $\mathrm{ng} \mathrm{m}^{-3}$ for $\mathrm{BC}$ and $\mu \mathrm{g} \mathrm{m}^{-3}$ for

5 PM. Pollutant sensor: measured pollutant and sensor's name; $M$ : mean concentration; Match:

6 match score; RMSE: root mean squared error; $r$ : Pearson correlation coefficient; $t$ : Kendall

7 correlation coefficient; S: Spearman correlation coefficient; Pres: presence parameter; LFE: low

8 frequencies energy parameter; IPI: SET integrated performance index; SensTB: sensor time base:

9 RefTB: reference time base; Start time: measurement's beginning; End time: measurement's

10 ending. 
14 Table 1. Tested sensor specifications.

\begin{tabular}{|c|c|}
\hline \multicolumn{2}{|c|}{ Expected specifications } \\
\hline \multirow{4}{*}{ Measurement range } & $\mathrm{O}_{3}=[0 ; 250] \mathrm{ppb}$ \\
\hline & $\mathrm{NO}_{2}=[0 ; 500] \mathrm{ppb}$ \\
\hline & $\mathrm{BC}=[0 ; 50000] \mathrm{ng} \mathrm{m}^{-3}$ \\
\hline & $\mathrm{PM}_{10}$ and $\mathrm{PM}_{2.5}=[0 ; 1000] \mu \mathrm{g} \mathrm{m}^{-3}$ \\
\hline & VOC $=$ depending on the sensor specificity ability \\
\hline Time step & below $5 \mathrm{~min}$ \\
\hline Battery life & 12 hours as a minimum \\
\hline Temperature range & {$[-10 ; 40]^{\circ} \mathrm{C}$} \\
\hline Weight (total to be worn) & below $2 \mathrm{~kg}$ \\
\hline Detection limit, precision & To be specified by the manufacturer \\
\hline
\end{tabular}

15 
17 Table 2. Main expected specifications of the sensors.

\begin{tabular}{cccc}
\hline Sensor & Price $(€)$ & Weight $(\mathrm{g})$ & Measured pollutant \\
\hline A & $5000-10000$ & $200-400$ & $\mathrm{BC}$ \\
B & $2000-5000$ & $600-800$ & $\mathrm{PM}_{10}, \mathrm{NO}_{2}, \mathrm{O}_{3}, \mathrm{VOC}$ \\
C & $500-1000$ & $0-100$ & $\mathrm{NO}_{2}$ \\
D & $1000-2000$ & $400-600$ & $\mathrm{NO}_{2}$ \\
E & $2000-5000$ & $600-800$ & $\mathrm{PM}_{10}, \mathrm{PM}_{2.5}, \mathrm{PM}_{1}$ \\
F & $500-1000$ & $600-800$ & $\mathrm{PM}_{10}, \mathrm{PM}_{2.5}, \mathrm{PM}_{1}$ \\
G & $1000-2000$ & $100-200$ & $\mathrm{VOC}$ \\
$\mathrm{H}$ & $2000-5000$ & $400-600$ & $\mathrm{CO}, \mathrm{NO}, \mathrm{NO}_{2}, \mathrm{O}_{3}, \mathrm{PM}_{10}$ \\
\hline
\end{tabular}

18

19 


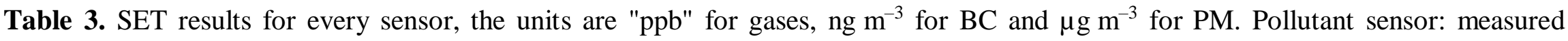
pollutant and sensor's name; $M$ : mean concentration; Match: match score; RMSE: root mean squared error; $r$ : Pearson correlation coefficient; $t$ : Kendall correlation coefficient; $S$ : Spearman correlation coefficient; Pres: presence parameter; LFE: low frequencies energy parameter; IPI: SET integrated performance index; SensTB: sensor time base: RefTB: reference time base; Start time: measurement's beginning; End time: measurement's ending.

\begin{tabular}{|c|c|c|c|c|c|c|c|c|c|c|c|c|c|}
\hline $\begin{array}{c}\text { Pollutant } \\
\text { sensor }\end{array}$ & $M$ & Match & RMSE & $r$ & $t$ & $S$ & Pres & LFE & IPI & $\begin{array}{c}\text { SensTB } \\
\text { (min) }\end{array}$ & $\begin{array}{c}\text { RefTB } \\
(\min )\end{array}$ & Start time (TU) & End time (TU) \\
\hline $\mathrm{BC} \mathrm{A}$ & 1077 & 0.88 & 268 & 0.98 & 0.85 & 0.96 & 0.97 & 1.00 & 0.91 & 5 & 1 & $23 / 01 / 2017$ 07:55 & $06 / 02 / 2017$ 15:05 \\
\hline $\mathrm{O}_{3} \mathrm{~B}$ & 8 & 0.30 & 15 & 0.70 & 0.60 & 0.80 & 0.75 & 1.00 & 0.46 & 1 & 1 & 23/01/2017 07:43 & 03/02/2017 08:44 \\
\hline $\mathrm{NO}_{2} \mathrm{~B}$ & 11 & 0.46 & 5 & 0.94 & 0.72 & 0.89 & 0.78 & 1.00 & 0.76 & 1 & 1 & 23/01/2017 07:43 & 03/02/2017 08:44 \\
\hline $\mathrm{NO}_{2} \mathrm{C}$ & 20 & 0.35 & 13 & 0.04 & 0.08 & 0.12 & 1.00 & 1.00 & 0.42 & 1 & 1 & 20/01/2017 14:00 & 27/01/2017 10:00 \\
\hline $\mathrm{NO}_{2} \mathrm{D}$ & 47 & 0.24 & 37 & 0.54 & 0.54 & 0.73 & 0.67 & 1.00 & 0.56 & 1 & 1 & 23/01/2017 13:56 & 01/02/2017 10:09 \\
\hline $\mathrm{PM}_{10} \mathrm{~B}$ & 104 & 0.43 & 112 & 0.18 & 0.20 & 0.26 & 0.81 & 0.99 & 0.40 & 1 & 15 & 23/01/2017 07:45 & 03/02/2017 08:45 \\
\hline $\mathrm{PM}_{10} \mathrm{E}$ & 535 & 0.37 & 1819 & 0.06 & 0.38 & 0.52 & 0.69 & 0.89 & 0.07 & 1 & 15 & 23/01/2017 08:30 & 03/02/2017 16:00 \\
\hline $\mathrm{PM}_{10} \mathrm{~F}$ & 21 & 0.63 & 16 & 0.84 & 0.33 & 0.46 & 1.00 & 0.99 & 0.64 & 1.5 & 15 & $26 / 01 / 201712: 30$ & $06 / 02 / 201715: 15$ \\
\hline $\mathrm{PM}_{2.5} \mathrm{E}$ & 136 & 0.43 & 185 & 0.45 & 0.52 & 0.72 & 0.69 & 0.99 & 0.49 & 1 & 15 & 23/01/2017 08:30 & 03/02/2017 16:00 \\
\hline $\mathrm{PM}_{2.5} \mathrm{~F}$ & 18 & 0.76 & 10 & 0.91 & 0.66 & 0.82 & 1.00 & 0.99 & 0.80 & 1.5 & 15 & 26/01/2017 12:30 & 06/02/2017 15:15 \\
\hline $\mathrm{PM}_{1} \mathrm{E}$ & 43 & 0.43 & 33 & 0.76 & 0.62 & 0.82 & 0.69 & 1.00 & 0.65 & 1 & 15 & 23/01/2017 08:30 & 03/02/2017 16:00 \\
\hline $\mathrm{PM}_{1} \mathrm{~F}$ & 13 & 0.77 & 8 & 0.88 & 0.66 & 0.82 & 1.00 & 0.99 & 0.78 & 1.5 & 15 & 26/01/2017 12:30 & $06 / 02 / 201715: 15$ \\
\hline
\end{tabular}


2 Fig. 1. Routes for the mobility tests (plotted in red and blue). Sources: actualitix, and

3 OpenStreetMap (modified).

4 Fig. 2. $\mathrm{BC}$ and $\mathrm{NO}_{2}$ time series in static measurements.

5 Fig. 3. Mobility tests for $\mathrm{NO}_{2}$ sensors. The three sensors $\mathrm{C}$ are plotted in shades of green, the

6 three sensors B in shades of orange, the reference Airparif stations are plotted in black.

7 Fig. 4. Mobility tests for $\mathrm{PM}_{10}$ sensors. The three sensors $\mathrm{F}$ are plotted in shades of blue, the three

8 sensors B in shades of orange, the reference Airparif stations are plotted in black.

9 Fig. 5. Reproducibility tests for sensors A, C and F with reference instruments.

10 Fig. 6. Chamber controlled tests for $\mathrm{BC}$ sensor $\mathrm{A}, \mathrm{PM}$ sensor $\mathrm{F}$ and $\mathrm{NO}_{2}$ sensor $\mathrm{C}$.

11 Fig. 7. Mobility test results. 


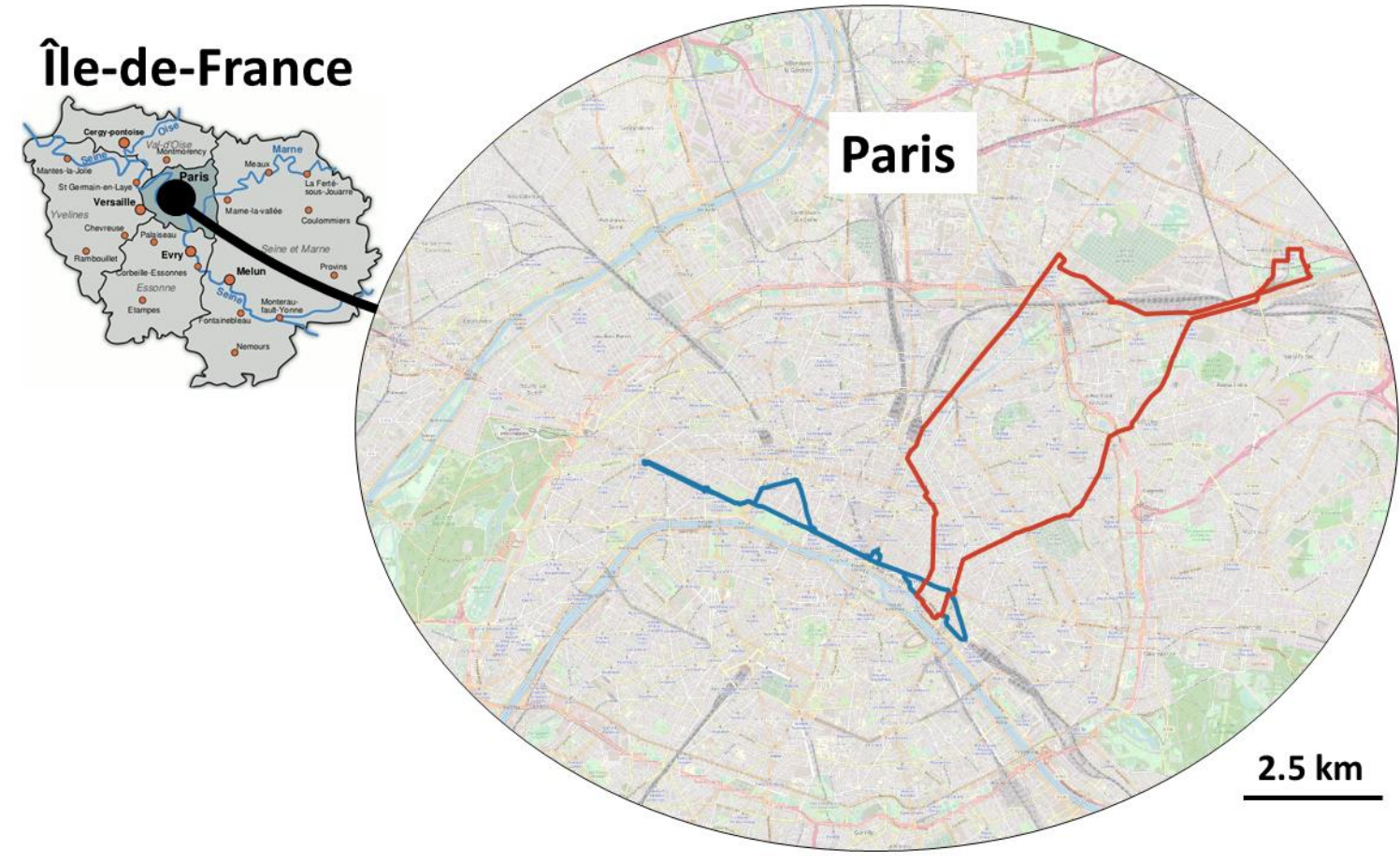



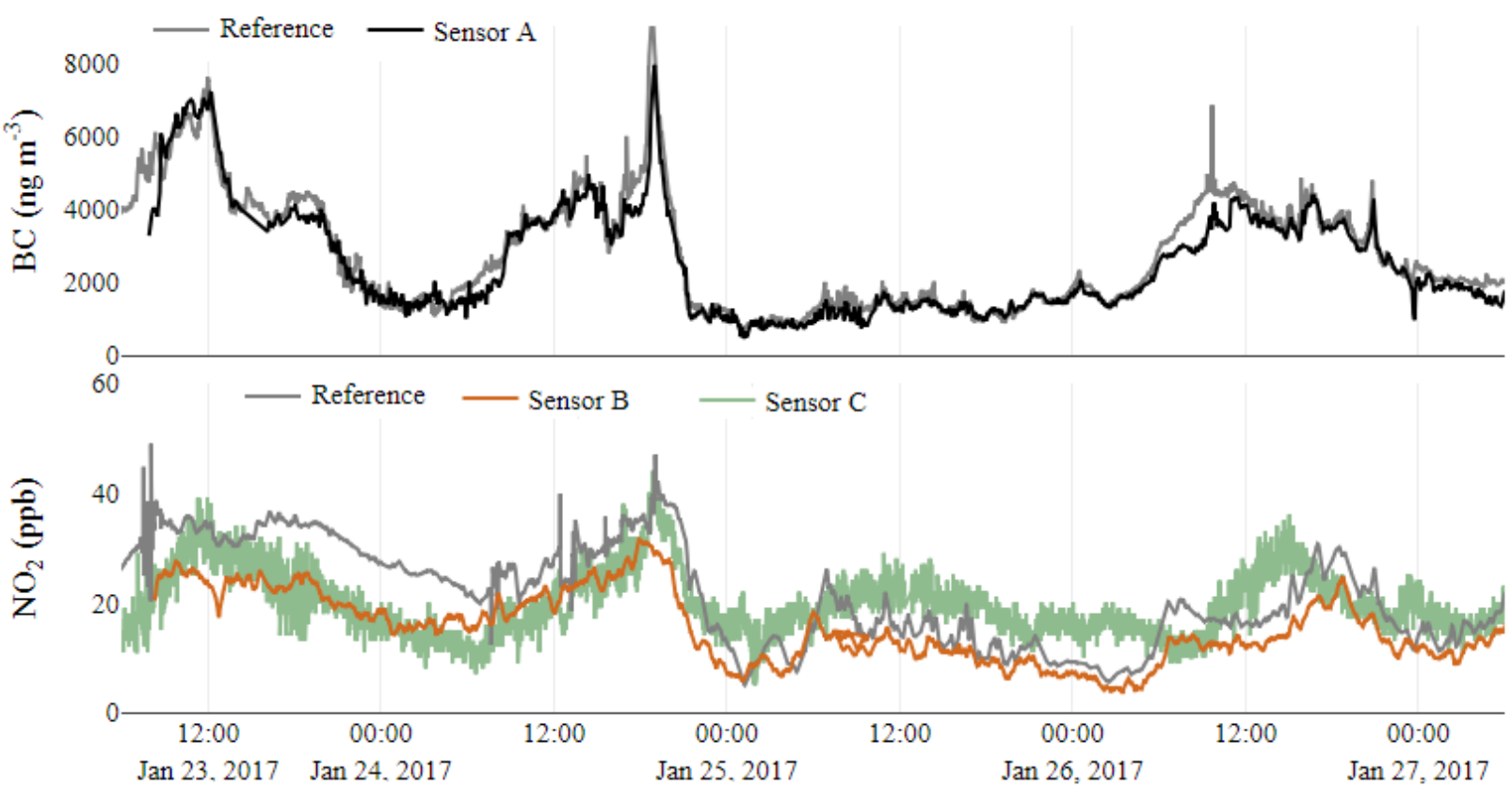

Fig. 2. 
23
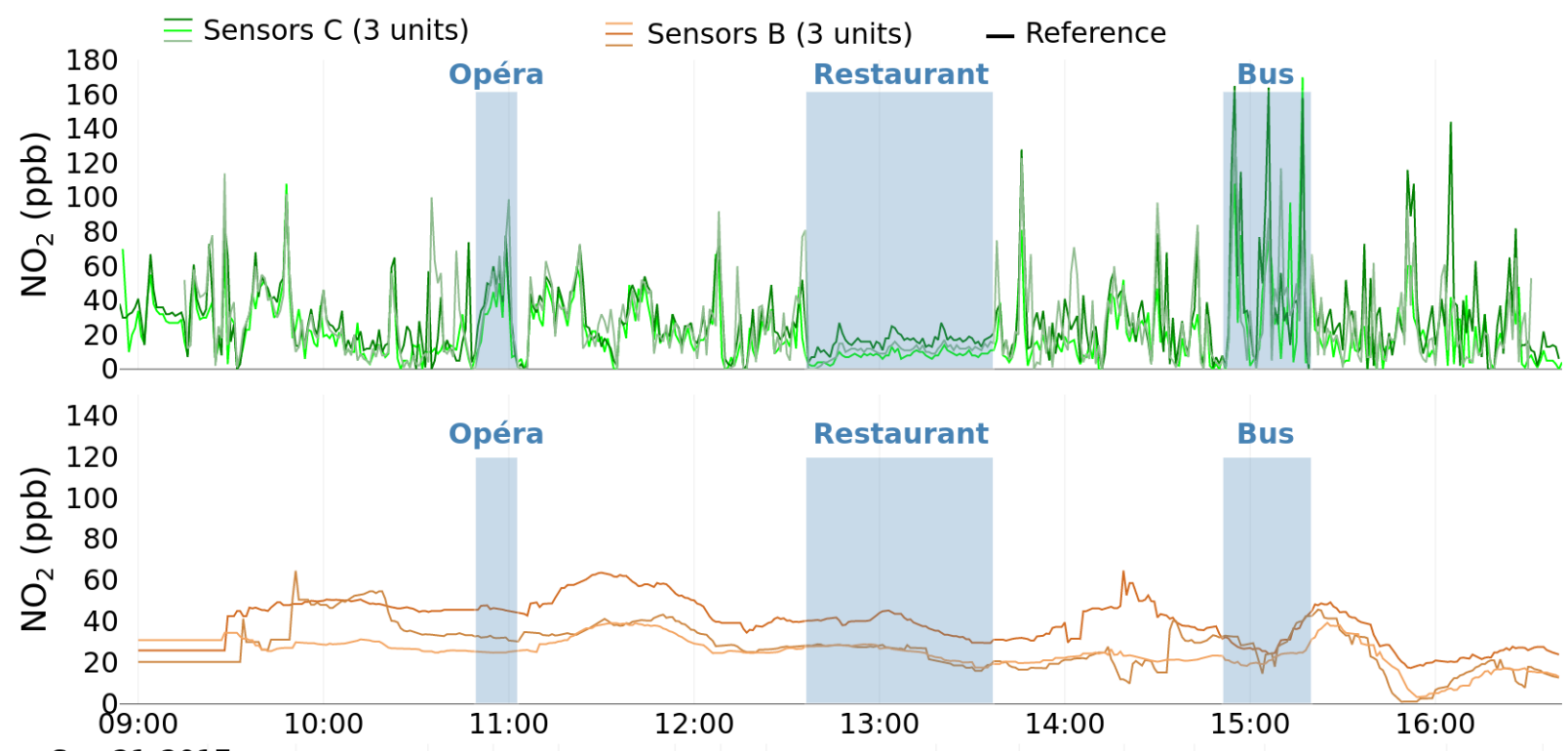
Sep 21,2017

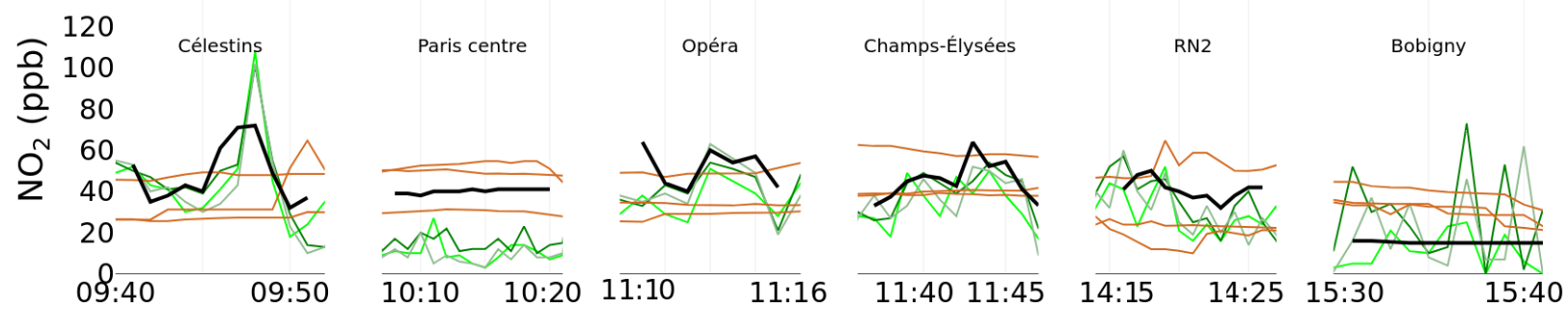

Fig. 3. 
28

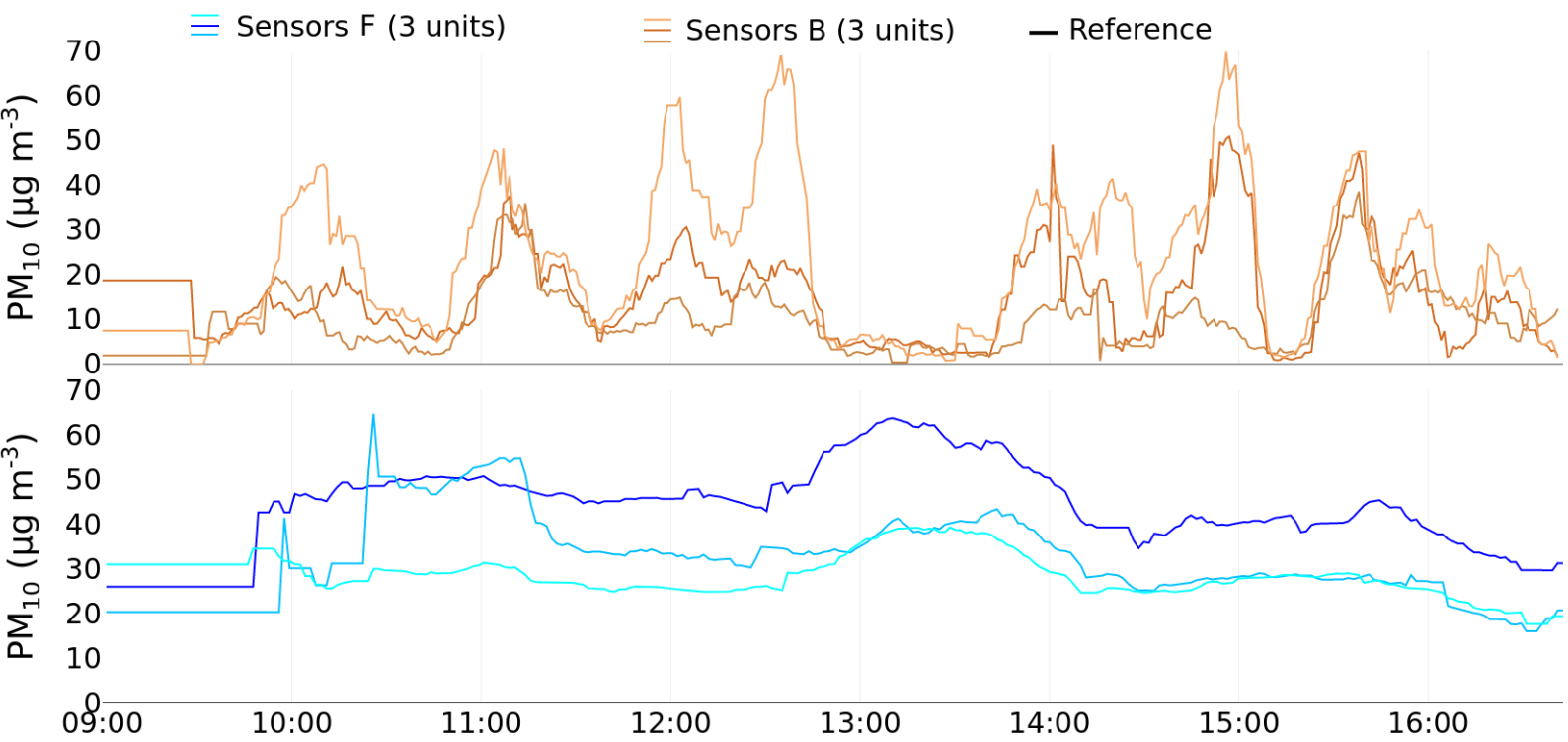

Sep 21, 2017

m $60 \quad$ Paris centre

E 50

을 40

$\sum_{0}^{\circ} 20$

$\sum_{\Delta} 10$

29
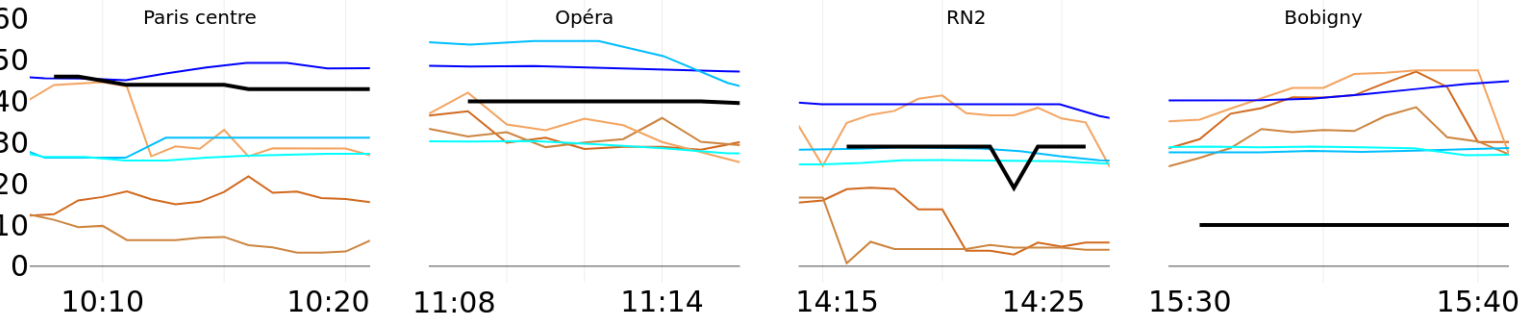

30

31

Fig. 4.

32 

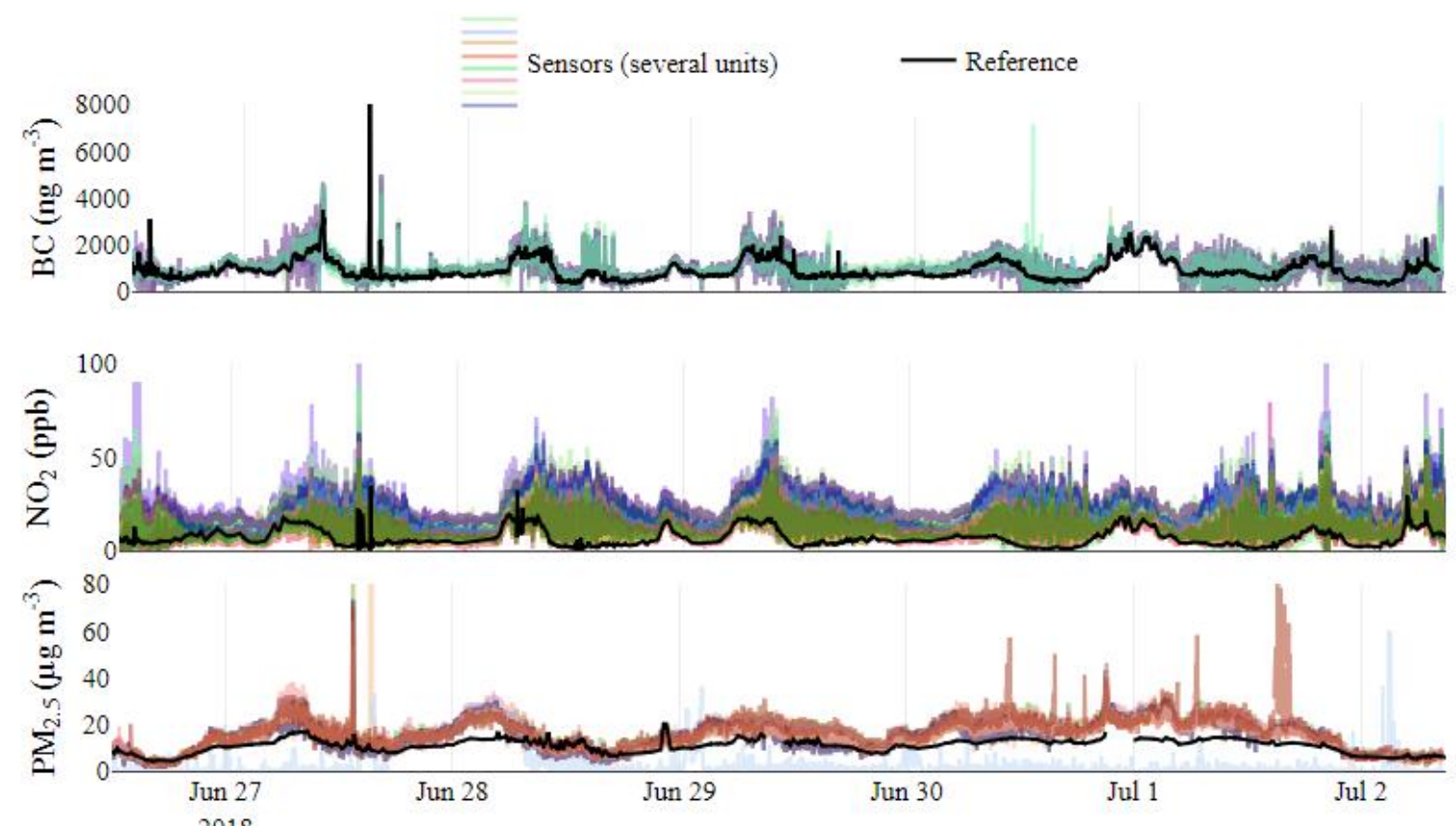

34

Fig.5.

36 

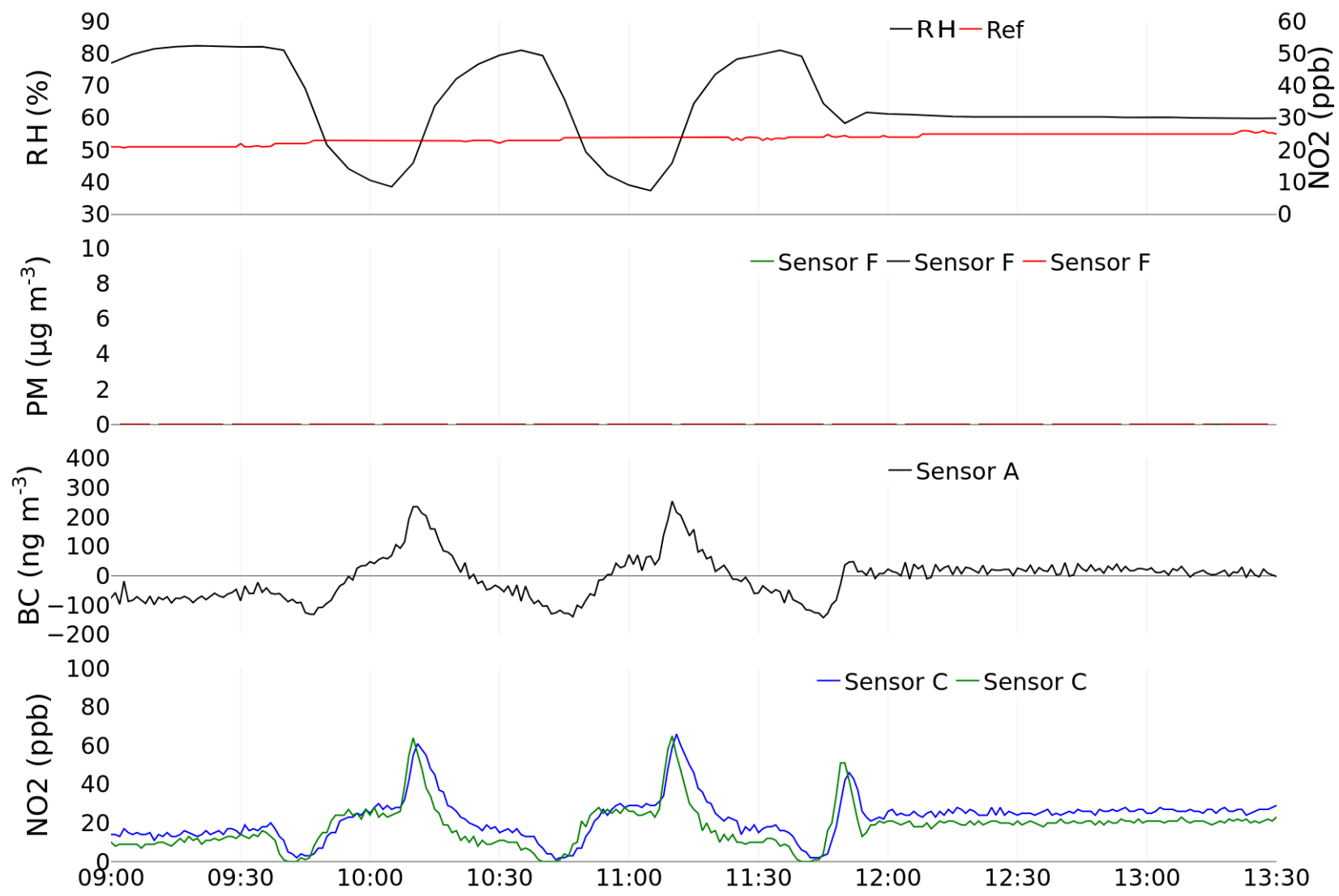
Jul 3, 2018 

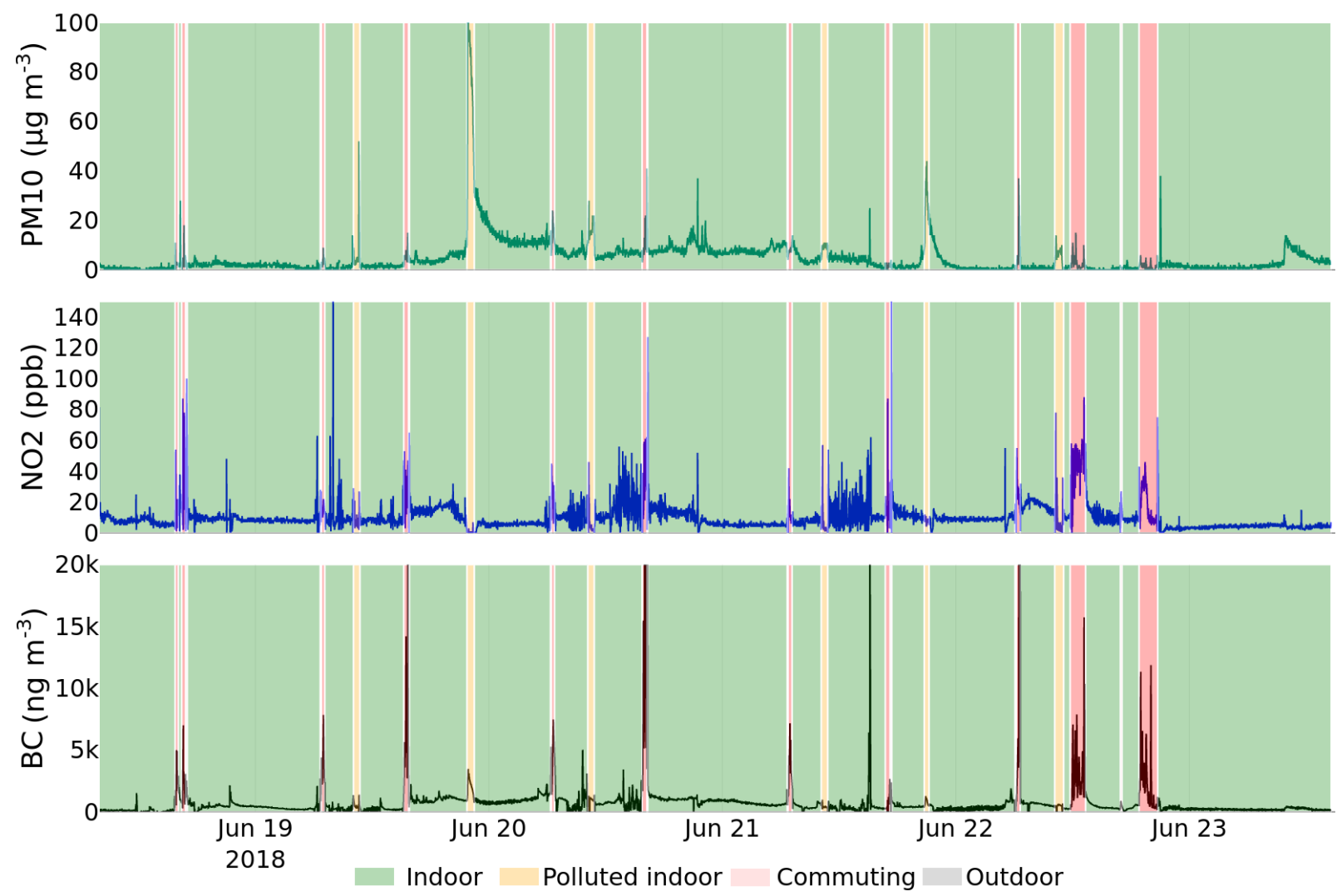

Fig. 7. 